\title{
Evaluation of CDK6 and p16/INK4a-Derived Peptides Interaction
}

\author{
Andrey Kazennov ${ }^{1}$, Andrey Alekseenko ${ }^{1}$, Vladimir Bozhenko ${ }^{2}$, \\ Tatiana Kulinich $^{2}$, Nikolay Shuvalov ${ }^{1}$, Yaroslav Kholodov ${ }^{1}$ \\ ${ }^{1}$ Department of Computational Mathematics, Moscow Institute of Physics and Technology, \\ Dolgoprudny, Russia \\ ${ }^{2}$ Russian Scientific Center of Roentgenoradiology, Moscow, Russia \\ Email: kazennov@gmail.com
}

Received May 22, 2013; revised June 22, 2013; accepted June 30, 2013

Copyright (C) 2013 Andrey Kazennov et al. This is an open access article distributed under the Creative Commons Attribution License, which permits unrestricted use, distribution, and reproduction in any medium, provided the original work is properly cited.

\begin{abstract}
The goal of this work is the development of novel peptides with high efficacy of inhibiting activity of CDK6/CyclinD complex. The peptides were derived from primary sequence of P16 protein and its homologues. The interactions between CDK6 and P16/INK4a-derived peptides are studied with molecular dynamics simulation employing umbrella sampling method. The SASA implicit solvent model was used for simulation, which was accelerated using NVIDIA GPUs.
\end{abstract}

Keywords: Cyclin-Dependent Kinases; Molecular Dynamics; Umbrella Sampling; GPU; SASA

\section{Introduction}

Cyclin-dependent kinases (CDKs) play a major role in cell cycle regulation and cell division progression. The correct performance of various cyclin-dependent kinases secures the sequential progression of numerous metabolic processes required for cell division. CyclinD-activated kinases CDK4 and CDK6 are quintessential for cell progression from growth phase $\left(\mathrm{G}_{1}\right)$ to synthesis phase (S). The CyclinD-CDK4/6 complex activity is regulated by proteins of CKI (Cyclin-dependent kinase inhibitors) family. Many pathological processes including malignant tumors of various locations associated with abnormalities in cell division process are caused by defects in CyclinD-CDK4/6 complex functioning, which are conditioned by hyperexpression of CyclinD or CDK$4 / 6$, mutation of intracellular inhibitors or a number of other processes. CDK4 and CDK6 are attractive molecular targets since it was shown that pharmacological inhibition of CDK4/6 leads to growth suppression in tumors having declension in CDK4/6-involving control pathway.

Currently, numerous low-molecular CDK inhibitors are known [1]. However, their major disadvantage is low selectivity since they are mimicking ATP and are competing with it for binding sites, which are homologous in most kinases, thus the problem of cross-reactivity arises
[2]. More preferable from our point of view is the use of peptides derived from natural CKI proteins. In vivo and in vitro experiments have shown that short (up to 10 residues) peptides from p16/INK4a, p18/INK4c, p21/ WAF1 and $27 /$ Kip 1 proteins have inhibitory properties comparable with activity of full-sized protein [3]. The use of peptides as pharmaceutical intracellular drugs became possible after discovery advancement of "cell penetrating peptides" (CPP) technology [4], allowing delivery of almost-arbitrary peptide sequence into intracellular compartments with nearly $100 \%$ efficacy. The combination of CPP technology with highly selective functional sequences derived from natural regulatory proteins appears to be a promising approach for development of targeted drugs.

Numerical modeling is routinely used as a way to lower the drug developments costs by significantly reducing the number of potential pharmaceutical compounds. However there is no mastered methodology for evaluation of peptide drugs.

In this work protein docking and molecular dynamics methods are applied to the problem of predicting the efficacy of given peptides. The CDK6 protein is used as drug target, and the tested peptides were derived from p16/INK4a protein sequence. 


\section{Numerical Model}

To calculate the free energy of protein-peptide interaction we use SASA implicit solvent model implemented in GPU-accelerated MDis package [5]. Since the main task is to bolt out the most of a priory ineffective peptides the use of such simplified model is justified.

MDis package implements a CHARMM19 forcefield [6] in conjunction with SASA implicit solvent model [79].

\section{Biological System}

The major role in cell division control pathway is played by Retinoblastoma protein (pRb) (Figure 1). In activated (non-phosphorylated) state this protein suppresses cell cycle progression by inhibiting E2F transcription factor. In healthy cell ready for division, the phosphorylation (deactivation) of $\mathrm{pRb}$ is performed by complex of cyclindependent kinases CDK4 and CDK6 with cyclinD proteins (D1, D2, D3). The cancer cells often exhibit the mutations in one or more proteins involved in this pathway [10-14]. The natural inhibitors of CDK4 and CDK6 bound to CyclinD are proteins of INK4 family, including P16/INK4a protein, whose functioning is also known to be disrupted in cancerous cell $[11,12]$. The introduction of P16 protein into cell has been shown to arrest cell cycle progression from $\mathrm{G}_{1}$ to $\mathrm{S}$ phase.

The clinical use of P16, however, is not practical due to its relatively large size (156 amino acid residues). It might be more practical to develop shorter (up to 10 amino acid residues long) peptides with inhibitory activity on level with full-length P16.

As a foundation for this study we used the works of Fåhraeus et al. $[15,16]$ measuring in vitro and in vivo the efficiency of CDK6 and CDK4 inhibition by various

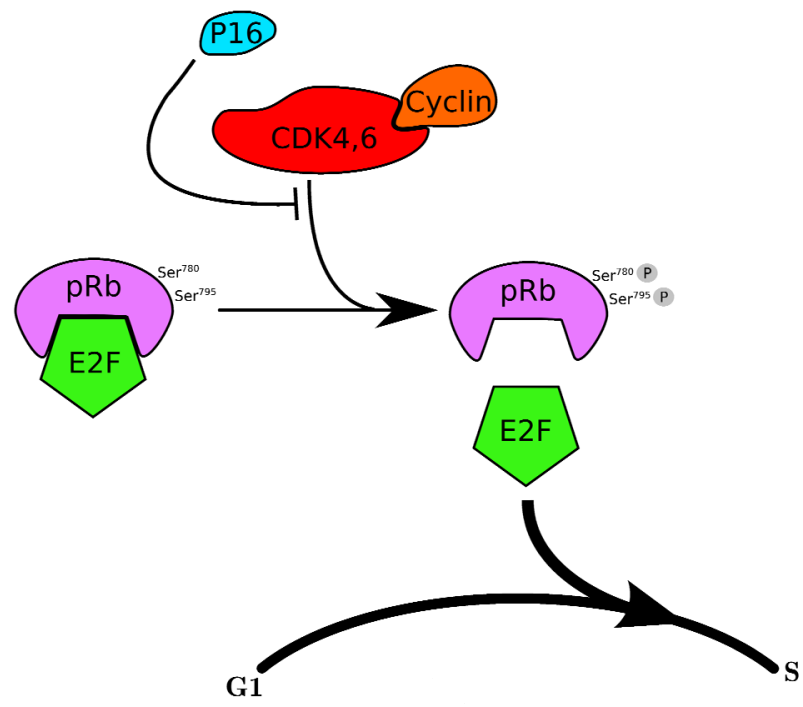

Figure 1. CDK4/6 regulatory pathway schematics. parts of P16 protein and its homologues.

The first series of experiments [15] measures the stability of complex formed by CDK6 and 20-residue-long peptides derived from all subsequences of P16 with 15residue step. Afterwards, the activity of CDK6 was measured in vitro in presence of same peptides, as well as their effect on cell cycle progression in vivo. In all of these experiments the peptide codenamed " $p 6$ " (residues 84 - 103 of full-sized P16) have shown best results.

Reference [16] describes the experiments measuring the efficiency of various mutations of "p6" peptide, including its shortening.

The main goal of this work is to reproduce the results of $[15,16]$ in silico to approbate the suggested protocol.

\section{Methodology}

The proteins of INK4 family are allosteric inhibitors [17, 18]. However, the characteristic times of structural transitions in proteins are of order of milliseconds which is beyond what is currently reachable by all-atom molecular dynamics simulations. So an assumption was made that the stable complex formation is required for peptide inhibitory activity since otherwise it would be unable to cause sufficient structural change. For this reason the energy profiles of association of CDK6 with peptides were studied.

Initial protein structures were taken from Protein Data Bank (CDK6: 1BLX [19], P16: 1BI7 [20]). Each structure was minimized with steepest-descend algorithm in MDis package.

In this work peptides codenamed P2-P9 from [15] are studied. The structure of peptides was obtained by cutting them from 3D structure of whole protein and subsequent equilibration for $5 \mathrm{~ns}$ at $300 \mathrm{~K}$ temperature.

Initial conformations of peptides bound to CDK6 were obtained using Autodock Vina software [21]. For each peptide ten most energetically favorable conformations were chosen, from which from 2 to 4 significantly different ones were chosen for further investigation.

To measure interaction energy the umbrella sampling technique [22] was used. The distance between peptide and protein centers of mass was taken as reaction coordinate. For each conformation in consideration the generation of initial trajectory was performed by pulling peptide apart from the protein with force applied to peptides center of mass and $\mathrm{C}_{\alpha}$ atoms of CDK6 restrained (Langevin integrator with $T=300 \mathrm{~K}, \gamma=0.15, \Delta t=1 \mathrm{fs}$, harmonic restraint on $\mathrm{C}_{\alpha}$ atoms of CDK6 $16 \mathrm{kcal} / \mathrm{mol} / \AA^{2}$, pulling speed $5 \AA / \mathrm{ns}$, pulling spring constant $2 \mathrm{kcal} / \mathrm{mol} /$ $\AA^{2}$ ). The trajectories were used to extract initial conformations for further sampling. The conformations were taken with $0.5 \AA$ step of reaction coordinate. For each sampling window the $30 \mathrm{~ns}$ of equilibration simulation 
was performed with additional harmonic restraint applied to reaction coordinate (Langevin integrator with $T=300$ $\mathrm{K}, \gamma=0.15, \Delta t=1 \mathrm{fs}$; umbrella spring constant $1 \mathrm{kcal} /$ $\mathrm{mol} / \AA^{2}$; restraint on $\mathrm{C}_{\alpha}$ atoms of CDK6 $2.4 \mathrm{kcal} / \mathrm{mol} / \AA^{2}$ ).

After analyzing resulting trajectories with weighted histogram analysis method (WHAM) [23] the free energy profiles were obtained. We used implementation of WHAM by A. Grossfield [24].

The free energy difference between bound state and the highest point of energy barrier separating bound and unbound states were chosen as resulting value for each peptide studied.

\section{Results and Discussion}

The conformations obtained from protein docking study are shown in Figure 2, and the primary sequences of peptides are listed in the Table 1. As one can see, all peptides prefer hydrophobic pockets between two lobes of CDK6 which coheres with proposed structural model of CDK4/6 inhibition by proteins of INK4 family [17].

Figures 3 and $\mathbf{4}$ show the obtained results for binding energy compared to in vitro results from $[15,16]$. As could be seen in Figure 4 although numerical modeling yields correct interaction energy it is not always directly related to inhibition efficiency.

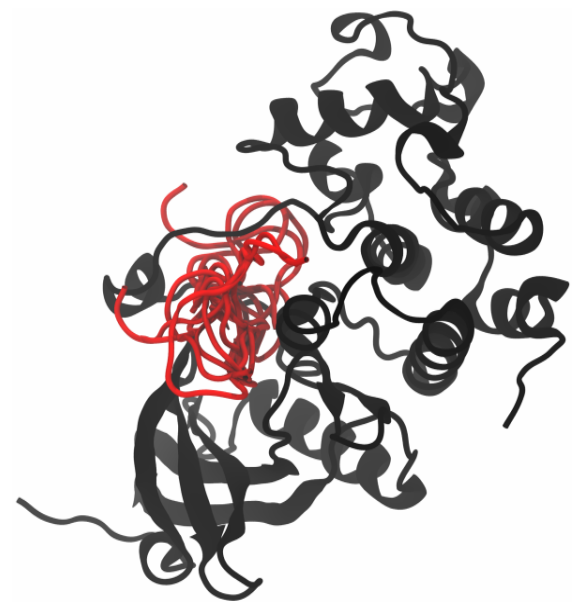

Figure 2. CDK6 (black) and peptides (red) bound conformations as predicted by Autodock Vina [21].

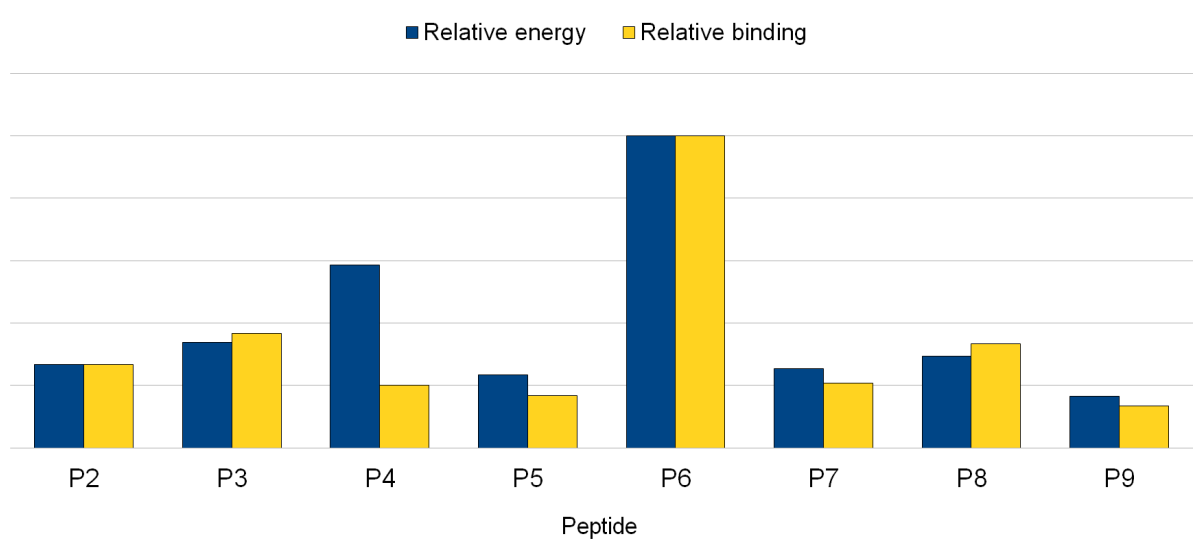

Figure 3. The comparison of in silico (blue) and in vitro (yellow) results for the first series of experiments. For in silico, the energy of binding obtained with Umbrella sampling is shown. For in vitro, the data on relative binding on agarose gel is shown.

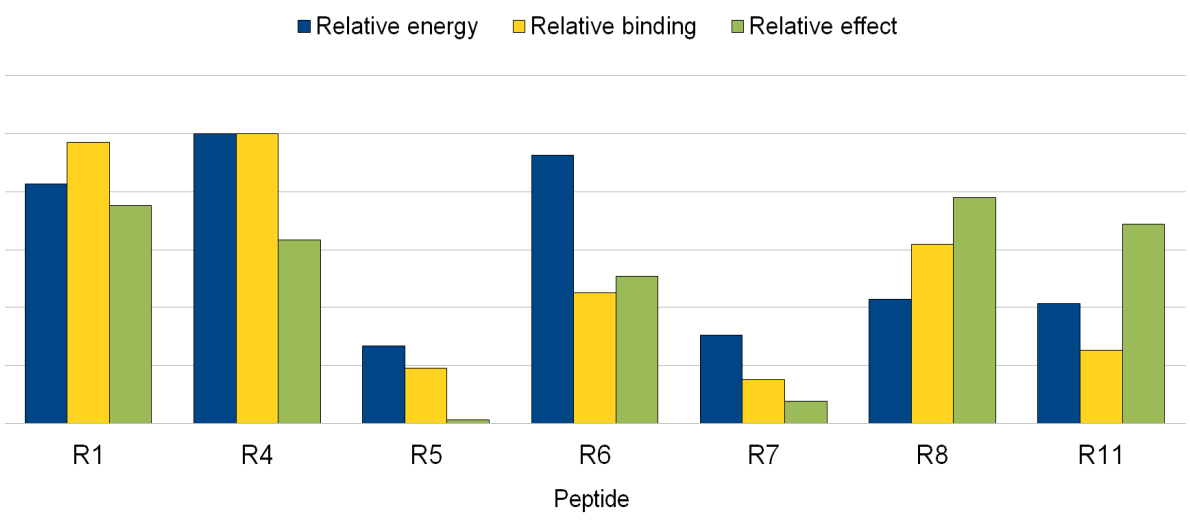

Figure 4. The comparison of in silico (blue) and in vitro (yellow, green) results for the second series of experiments. For in silico, the energy of binding obtained with Umbrella sampling is shown. For in vitro, the data on relative binding on agarose gel (yellow) and relative kinase activity in presence of studied peptide (green) is shown. 
Table 1. Studied peptides.

\begin{tabular}{ccc}
\hline Peptide & Sequence & Source \\
\hline P2 & RVEEVRALLEAGANPNAPNS & {$[15]$} \\
P3 & NAPNSYGRRPIQVMMMGSAR & {$[15]$} \\
P4 & MGSARVAELLLLHGAEPNCA & {$[15]$} \\
P5 & EPNCADPATLTRPVHDAARE & {$[15]$} \\
P6 & DAAREGFLDTLVVLHRAGAR & {$[15]$} \\
P7 & RAGARLDVRDAWGRLPVDLA & {$[15]$} \\
P8 & PVDLAEELGHRDVARYLRAA & {$[15]$} \\
P9 & YLRAAAGGTRGSNHARIDAA & {$[15]$} \\
P10 & DAARAGFLDTLQTLLEFQAD & {$[15]$} \\
P11 & DAAREGFLDTLVVLHRAGAR & {$[15]$} \\
R1 & DAAREGFLDTLVVLHRAG & {$[16]$} \\
R4 & DAAREGFLDTLVVLHR & {$[16]$} \\
R5 & AREGFLDTLVVLHRAGAR & {$[16]$} \\
R6 & EGFLDTLVVLHRAGAR & {$[16]$} \\
R7 & FLDTLVVLHR & {$[16]$} \\
R8 & RVEEVRALLEAGANPNAPNS & {$[16]$} \\
R11 & NAPNSYGRRPIQVMMMGSAR & {$[16]$} \\
\hline
\end{tabular}

\section{Conclusion}

The result of this work is the approbation of methodology for numerical evaluation of binding energy of given peptides with chosen target protein. The results could be improved at the expense of increased simulation wall time by using more accurate implicit solvent models (e.g. GB/SA [25] or FACTS [26]) or explicit solvent. Alternative approach to improve the accuracy is to use more initial conformations.

\section{Acknowledgements}

The work was supported by grants from Ministry of Science and Education of Russian Federation \#14.A18.21. 1871 and \#14.A18.21.1239.

The "Lomonosov" supercomputer installed in Supercomputing complex of Moscow State University was used to perform simulations.

\section{REFERENCES}

[1] A. Hughes, "CDK Inhibitors in 3D: Problems with the Drugs, Their Development Plans or Their Linkage to Disease?" Gene therapy \& Molecular Biology, Vol. 10, 2006, pp. 41-54.

[2] O. Fedorov, B. Marsden, V. Pogacic, P. Rellos and S.
Müller, "A Systematic Interaction Map of Validated Kinase Inhibitors with Ser/Thr Kinases," Proceedings of the National Academy of Sciences of the United States of America, Vol. 104, No. 51, 2007, pp. 20523-20528. doi:10.1073/pnas.0708800104

[3] V. P. Kharchenko, V. K. Bojenko, T. M. Kulinich, V. G. Lunin, Ye. I. Filyasova, A. M. Shishkin, O. V. Sergueenko, Ye. M. Ryazanova and O. L. Voronina, "Cytotoxity of Chimera Peptides Incorporating Sequences of Cycline Kinases Inhibitors," Voprosy Oncologii, Vol. 53, No. 4, 2007, pp. 448-452.

[4] M. Lindgrena, M. Hällbrinka, A. Prochiantzb and Ü. Langela, "Cell-Penetrating Peptides," Trends in Pharmacological Sciences, Vol. 21, No. 3, 2000, pp. 99-103. doi:10.1016/S0165-6147(00)01447-4

[5] A. Zhmurov, O. Kononova, R. I. Litvinov, R. I. Dima, V. Barsegov and J. W. Weisel, "Mechanical Transition from $\alpha$-Helical Coiled Coils to $\beta$-Sheets in Fibrin(ogen)," Journal of the American Chemical Society, Vol. 134, No. 50, 2012, pp. 20396-20402. doi:10.1021/ja3076428

[6] B. R. Brooks, R. E. Bruccoleri, B. D. Olafson, D. J. States, S. Swaminathan and M. Karplus, "CHARMM: A Program for Macromolecular Energy, Minimization, and Dynamics Calculations," Journal of Computational Chemistry, Vol. 4, No. 2, 1983, pp. 187-217. doi: $10.1002 /$ jcc. 540040211

[7] P. Ferrara, J. Apostolakis and A. Caflisch, "Evaluation of a Fast Implicit Solvent Model for Molecular Dynamics Simulations," Proteins, Vol. 46, No. 1, 2002, pp. 24-33. doi:10.1002/prot.10001

[8] F. Fraternali and W. F. van Gunsteren, "An Efficient Mean Solvation Force Model for Use in Molecular Dynamics Simulations of Proteins in Aqueous Solution," Journal of Molecular Biology, Vol. 256, No. 5, 1996, pp. 939-948. doi:10.1006/jmbi.1996.0139

[9] D. Eisenberg and A. D. McLachlan, "Solvation Energy in Protein Folding and Binding," Nature, Vol. 319, 1986, pp. 199-203. doi:10.1038/319199a0

[10] P. W. Hinds, S. Mittnacht, V. Dulic, A. Arnold, S. I. Reed and R. A. Weinberg, "Regulation of Retinoblastoma Protein Functions by Ectopic Expression of Human Cyclins," Cell, Vol. 70, No. 6, 1992, pp. 993-1006. doi:10.1016/0092-8674(92)90249-C

[11] C. W. Miller, A. Aslo, M. J. Campbell, N. Kawamata, B. C. Lampkin and H. P. Koeffler, "Alterations of the p15, p16, and p18 Genes in Osteosarcoma," Cancer Genetics and Cytogenetics, Vol. 86, No. 2, 1996, pp. 36-142. doi:10.1016/0165-4608(95)00216-2

[12] W. H. Liggett and D. Sidransky, "Role of the p16 Tumor Suppressor Gene in Cancer," Journal of Clinical Oncology: official Journal of the American Society of Clinical Oncology, Vol. 16, No. 3, 1998, pp. 1197-1206.

[13] J. Newton Bishop, M. Harland, D. C. Bennett, V. Bataille, M. Goldstein, et al., "Mutation Testing in Melanoma Families: INK4A, CDK4 and INK4D," British Journal of Cancer, Vol. 80, No. 1-2, 1999, pp. 295-300. doi:10.1038/sj.bjc.6690354

[14] T. M. Becker, H. Rizos, R. F. Kefford and G. J. Mann, 
"Functional Impairment of Melanoma-Associated p16(INK4a) Mutants in Melanoma Cells Despite Retention of Cyclin-Dependent Kinase 4 Binding," Clinical Cancer Research: An Official Journal of the American Association for Cancer Research, Vol. 7, No. 10, 2001, pp. 32823288.

[15] R. Fåhraeus, J. M. Paramio, K. L. Ball, S. Laín and D. P. Lane, "Inhibition of pRb Phosphorylation and Cell-Cycle Progression by a 20-Residue Peptide from p16CDKN2/ INK4A," Current Biology, Vol. 6, No. 1, 1996, pp. 84-91. doi:10.1016/S0960-9822(02)00425-6

[16] R. Fåhraeus, S. Laín, K. L. Ball and D. P. Lane, "Characterization of the Cyclin-Dependent Kinase Inhibitory Domain of the INK4 Family as a Model for a Synthetic Tumour Suppressor Molecule," Oncogene, Vol. 16, No. 5, 1998, pp. 587-596. doi:10.1038/sj.onc. 1201580

[17] P. D. Jeffrey, "Structural Basis of Inhibition of CDKCyclin Complexes by INK4 Inhibitors," Genes \& Development, Vol. 14, No. 24, 2000, pp. 3115-3125. doi:10.1101/gad.851100

[18] N. P. Pavletich, "Mechanisms of Cyclin-Dependent Kinase Regulation: Structures of Cdks, Their Cyclin Activators, and Cip and INK4 Inhibitors," Journal of Molecular biology, Vol. 287, No. 5, 1999, pp. 821-828. doi:10.1006/jmbi.1999.2640

[19] D. H. Brotherton, V. Dhanaraj, S. Wick, L. Brizuela, P. J. Domaille, et al., "Crystal Structure of the Complex of the cyclin D-Dependent Kinase Cdk6 Bound to the Cell-Cycle Inhibitor p19INK4d," Nature, Vol. 395, No. 6699, 1998, pp. 244-250. doi:10.1038/26164

[20] A. A. Russo, L. Tong, J. O. Lee, P. D. Jeffrey and N. P. Pavletich, "Structural Basis for Inhibition of the CyclinDependent Kinase Cdk6 by the Tumour Suppressor p16INK4a," Nature, Vol. 395, No. 6699, 1998, pp. 237 243. doi: $10.1038 / 26155$

[21] O. Trott and A. J. Olson, “AutoDockVina: Improving the Speed and Accuracy of Docking with a New Scoring Function, Efficient Optimization, and Multithreading," Journal of Computational Chemistry, Vol. 31, No. 2, 2010, pp. 455-461. doi:10.1002/jcc.21334

[22] G. M. Torrie and J. P. Valleau, "Nonphysical Sampling Distributions in Monte Carlo Free-Energy Estimation: Umbrella Sampling," Journal of Computational Physics, Vol. 23, No. 2, 1977, pp. 187-199. doi:10.1016/0021-9991(77)90121-8

[23] S. Kumar, J. M. Rosenberg, D. Bouzida, R. H. Swendsen and P. A. Kollman, "The Weighted Histogram Analysis Method for Free-Energy Calculations on Biomolecules. I. The Method," Journal of Computational Chemistry, Vol. 13, No. 2, 1992, pp. 1011-1021. doi:10.1002/jcc.540130812

[24] A. Grossfield, "WHAM: The Weighted Histogram Analysis Method". http://membrane.urmc.rochester.edu/content/wham 